\title{
EXPLOITATION OF HYBRID VIGOUR IN CHERRY TOMATO
}

\author{
A. A. Hamed \\ Veg. Breed. Dept., Horticulture Res. Inst., Agric. Res. Center, Egypt.
}

Received: Apr. 13, 2017

Accepted: Apr. 27, 2017

\begin{abstract}
Nine diverse genotypes of cherry tomatoes (Solanum lycopersicum var. cerasiforme) were crossed to produce thirty six hybrids. All hybrids and their parents along with the commercial hybrid Katalina-522 were tested during winter seasons of 2014/15 and 2015/16 for their mean performance and heterosis based on high parent under greenhouse conditions at Kaha Vegetable Research Farm, Kaliobia Governorate, Horticulture Research Institute, Agriculture Research Center, Egypt. The performances of the produced $F_{1}$ hybrids indicated that there are some crosses showed significant high values for reasonable characters compared with the commercial hybrid. The crosses Ch $3 \times$ Ch 16, Ch $16 \times$ Ch 22, Ch $18 \times$ Ch 22 and Ch $22 \times$ Ch 25 exhibited significant highest values of yield/plant. Also, some $F_{1}$ hybrids showed significant heterosis based on high parent for some evaluated traits, viz., total yield/plant, fruit firmness, fruit flesh thickness, fruit TSS fruit and ascorbic acid content.
\end{abstract}

Key words: cherry tomato, hybrid, heterosis, yield.

\section{INTRODUCTION}

Cherry tomato (Solanum lycopersicum var. cerasiforme) is a botanical variety of the cultivated tomato. It is thought to be the ancestor of all cultivated tomatoes. The size of cherry tomatoes range from thumb tip to the size of a golf ball and can range from being spherical to slightly oblong in shape (Anonymous, 2009). It's a good source of vitamin C, solids content, good taste, antioxidant and fruit set even at high temperature (Kavitha et al 2014 and Renuka et al 2014). In recent years, the demand for cherry tomato has increased primarily due to the increase in quality, viz., appearance, flavor and nutrition (Alarcon et al 1994). Flavor is generally determined by total soluble solids (TSS, \% Brix) and can be high in cherry tomatoes.

Heterosis or hybrid vigor is an important biological phenomenon referring to the manifested superiority of the $F_{1}$ hybrid resulting from the cross of genetically dissimilar parents. Heterosis describes the superior performance of heterozygous $\mathrm{F}_{1^{-}}$ hybrid plants in terms of increased biomass, size, yield, speed of development, fertility, resistance to disease and to insect pest, or to climatic rigours of any kind compared to the average of their homozygous parental inbred lines (Falconer and Mackay 1996). The terms high parent heterosis $(\mathrm{H})$ indicates that a hybrid trait performs significantly better than the higher of the two homozygous parental inbred lines.

Heterosis over high parent in cherry tomato was reported for the traits total yield, fruit weight, fruit length, fruit diameter, fruit firmness and fruit TSS by Salib (2012), for total yield, average fruit weight, fruit firmness and fruit ascorbic acid content by Mahmoud and El-Eslamboly (2014), for total yield by Muttappanavar et al (2014), for average fruit weight, fruit flesh thickness, fruit TSS and fruit ascorbic acid content by Pujer et al (2014), for total yield/plant, average fruit weight, fruit firmness and fruit thickness by Renuka et al (2015) and Renuka and Sadashiva (2016). Heterosis over control hybrid on cherry tomato was reported for the traits: total yield, average fruit weight, TSS and fruit ascorbic acid content (Fang et al 2002). Also, heterosis over standard hybrid in cherry tomato was reported for the traits total yield, average fruit weight, fruit firmness, fruit flesh thickness, fruit TSS and fruit ascorbic acid content (Khereba et al 2011). 
In Egypt, all the area of cherry tomato crop nowadays is still under $F_{1}$ hybrids which their seeds are imported from developed countries. At the same time little actual breeding efforts have been made for genetic improvement, as well as, for $F_{1}$ hybrid seeds production compared with that made for field crops. Therefore, there is urgent need for developing high yielding cherry tomato hybrids locally. The objective of the present study was to identify best cross combinations for developing promising cherry tomato hybrids for yield and fruit quality characters using nine diverse cherry tomato genotypes.

\section{MATERIALS AND METHODS}

This study was conducted during the period from 2013 to 2016 and involved production and evaluation of some $F_{1}$ cherry tomato hybrids. Crosses, transplant production and evaluations were carried out in the greenhouse at Kaha Vegetable Research Farm (KVRF), Kalubia Governorate, Horticulture Research Institute (HRI), Agriculture Research Center (ARC), Egypt. Nine diverse cherry tomato genotypes were chosen for their characters based on previous evaluation of available cherry tomato germplasm as shown in Table 1. The pure lines Ch 3, Ch 8, Ch 14, Ch 16,
Ch 18, Ch 21, Ch 22 and Ch 25 were derived through a breeding program of cherry tomato, HRI, ARC, Egypt (AboHamda 2012), meanwhile, the genotype Tomato 139 were derived from Heirloom Seed Project, Germany. Crosses were conducted among chosen 9 genotypes under greenhouse condition during the 2013/14 winter season to produce $36 F_{1}$ hybrids.

The produced $36 \quad F_{1}$ hybrids and their nine parents were evaluated along with the hybrid Katalina-522 as a control in the two successive winter plantings of 2014/15 and 2015/16 under greenhouse conditions. Seeding and transplanting dates were, respectively, last week of August and last week of September in both two seasons. A randomized complete blocks design (RCBD) with 3 replicates was used. Area of greenhouses was divided into 4 rows. Each row was $1.75 \mathrm{~m}$ wide and plants were transplanted on both sides of the row. The distance between plants, on each side of the row, was $40 \mathrm{~cm}$ apart, each experimental plot area was $3.5 \mathrm{~m}^{2}$ and consisted of 10 plants. The agricultural practices (fertilization, irrigation, and controlling weeds, diseases and insects) were performed as recommended for commercial tomato production in greenhouse.

Table 1. Genotypes of cherry tomato employed in the investigation.

\begin{tabular}{|llll|}
\hline Genotype & Parent & Source & Specific traits \\
\hline Ch 3 & $\mathrm{P}_{1}$ & L & $\begin{array}{l}\text { Determinate, low yRC, Egypt } \\
\text { dark red fruits, good firmness }\end{array}$ \\
\hline Ch $\mathbf{8}$ & $\mathrm{P}_{2}$ & HRI, ARC, Egypt & Determinate, dark red fruits \\
\hline Ch 14 & $\mathrm{P}_{3}$ & HRI, ARC, Egypt & Indeterminate, large yellow fruits \\
\hline Ch 16 & $\mathrm{P}_{4}$ & HRI, ARC, Egypt & Indeterminate, plant vigor, red fruits \\
\hline Ch 18 & $\mathrm{P}_{5}$ & HRI, ARC, Egypt & Indeterminate, large yellow fruits \\
\hline Ch 21 & $\mathrm{P}_{6}$ & HRI, ARC, Egypt & Determinate, large yellow fruits \\
\hline Ch 22 & $\mathrm{P}_{7}$ & HRI, ARC, Egypt & Indeterminate, red fruits \\
\hline Ch 25 & $\mathrm{P}_{8}$ & HRI, ARC, Egypt & $\begin{array}{l}\text { Indeterminate, plant vigor, dark red } \\
\text { fruits }\end{array}$ \\
\hline Tomato 139 & $\mathrm{P}_{9}$ & $\begin{array}{l}\text { Heirloom Seed Project, } \\
\text { Germany }\end{array}$ & $\begin{array}{l}\text { Indeterminate, high yielding, red } \\
\text { fruits }\end{array}$ \\
\hline Katalina-522 & $\begin{array}{l}\text { Check } \\
\text { hybrid }\end{array}$ & $\begin{array}{l}\text { GSI Seeds Company, USA } \\
\text { Indeterminate, plant vigor, high } \\
\text { yielding, red fruits, good firmness }\end{array}$ \\
\hline
\end{tabular}

\footnotetext{
${ }^{\mathrm{z}}$ HRI: Hort. Res. Institute, Agric. Res. Center, Egypt.
} 
Data were recorded on various evaluated genotypes for the traits: total yield per plant $(\mathrm{kg} /$ plant), average fruit weight $(\mathrm{g})$, fruit length $(\mathrm{mm})$, fruit diameter $(\mathrm{mm})$, fruit firmness $\left(\left(\mathrm{g} / \mathrm{cm}^{2}\right)\right)$, fruit flesh thickness $(\mathrm{mm})$, Total soluble solid (TSS \%) and ascorbic acid content (mg/100 $\mathrm{g}$ fresh fruit). Total yield was measured as the weight of all fruits harvested at the red ripe stage from each plot and the mean were taken for plant. Average fruit weight, fruit length and fruit diameter were determined as the mean of randomly 20 fruits/plot. Fruit firmness was measured in the red-ripe stage using a needle type pocket penetrometer. Five readings were taken for each fruit by pushing the pentrometer needle slowly at 5 different sites; one reading being near the shoulder, another one at the blossom end, and 3 readings at the equatorial plane, then mean of the 5 readings was calculated. Each plot was represented by randomly 20 fruits. Fruit flesh thickness was determined in a sample of 20 fruit per plot. Total soluble solid (TSS) was determined in at least 20 red-ripe fruits of each plot using a hand refractometer. Ascorbic acid content was determined using 2, 6 dichlorophenol indophenol dye (AOAC 1990).

Data obtained were statistically analyzed and mean comparisons were based on the LSD test (Gomez and Gomez 1984). Bartlett's tests of the variance of error for genotypes in both evaluated seasons were homogeneous for all traits. So, the combined analysis of variance for the two seasons was computed for all traits according to Snedecor and Cochran (1989).

Heterobeltiosis or high-parent heterosis $(\mathrm{HPH})$ was calculated in terms of percent increase $(+)$ or decrease (-) of the $F_{1}$ hybrids over its high parent as suggested by Fehr (1987).

$$
\mathrm{HPH}(\%)=\left[\left(\overline{F_{1}}-\overline{H P}\right) / \overline{H P}\right] * 100
$$

Where, $\overline{F_{1}}=$ mean of the hybrid for a specific trait and $\overline{H P}=$ mean of high parent in the cross.
Significance of high parent heterosis was determined following the " $\mathrm{t}$ " test.

\section{RESULTS AND DISCUSSION Total yield}

Combined analysis of both seasons (2014/15 and 2015/16 winter plantings) showed significant differences for total yield/plant among the evaluated genotypes (Table 2). Total yield/plant of the evaluated genotypes ranged from 0.905 to $6.001 \mathrm{~kg}$. The genotype $\mathrm{Ch} 22$ produced the highest total yield $(6.001 \mathrm{~kg} /$ plant $)$ among all evaluated genotypes followed by the genotype Ch 16 (5.752 kg/plant) with nonsignificant differences between them. Regarding hybrids, the hybrid Ch $18 \times \mathrm{Ch} 22$ gave the highest total yield $(5.718 \mathrm{~kg} / \mathrm{plant})$ followed by the hybrid Ch $3 \times$ Ch 16 (5.199 $\mathrm{kg} / \mathrm{plant}$ ) with non-significant differences between them but significant differences from the check hybrid Katalina-522 (4.667 $\mathrm{kg} /$ plant) was observed. For heterosis, three hybrids (Ch $3 \times$ Ch 8 , Ch $3 \times$ Ch 14 and Ch $14 \times \mathrm{Ch} 25)$ showed significant positive heterobeltiosis for total yield/plant, ranging from 49.2 to $69.4 \%$. These results are in harmony with those of Salib (2012), Mahmoud and El-Eslamboly (2014), Muttappanavar et al (2014), Renuka et al (2015) and Renuka and Sadashiva (2016) who found positive heterosis over high parent for this trait. Also, Fang et al (2002) and Khereba et al (2011) estimated heterosis over standard hybrid on cherry tomato for total yield.

\section{Average fruit weight}

Data obtained on average fruit weight trait of cherry tomato genotypes are presented in Table 3. Combined analysis of both seasons showed significant differences for this character among the evaluated genotypes, ranging from 9.7 to 22.4 gram. The highest average fruit weight was found in the genotype Ch $14(22.4 \mathrm{~g})$, followed by genotype Ch $21(21.7 \mathrm{~g})$ without significant difference between them. For hybrids, the hybrid Ch $14 \times$ Ch 21 had the heaviest fruits 
(20.7 g), followed by hybrid Ch $18 \times$ Ch 21 (19.3 g) with significant difference between them and with significant differences from the check hybrid Katalina-522 (11.1 g). The lowest value of average fruit weight was produced by the hybrids Ch $3 \times$ Ch 8 and Ch $3 \times$ Ch 25 (9.7 g). Concerning heterosis, none of the evaluated crosses showed a significant positive heterobeltiosis for average fruit weight trait. These results disagree with those found by Fang et al (2002), Khereba et al (2011), Salib (2012), Mahmoud and El-Eslamboly (2014), Pujer et al (2014), Renuka et al (2015) and Renuka and Sadashiva (2016). These different results could be due to using different genotypes or different environmental conditions.

Table 2. Mean performance of some cherry tomato genotypes and their $F_{1}$ 's and high parent heterosis $(\mathrm{H})$ for total yield/plant in the 2014/15 and 2015/16 winter plantings under greenhouse conditions.

\begin{tabular}{|c|c|c|c|c|c|c|c|c|c|}
\hline \multirow{2}{*}{ Genotype } & \multicolumn{4}{|c|}{ Total yield (kg/plant) } & \multirow{2}{*}{ Genotype } & \multicolumn{4}{|c|}{ Total yield (kg/plant) } \\
\hline & $2014 / 15$ & $2015 / 16$ & Mean & H (\%) & & $2014 / 15$ & $2015 / 16$ & Mean & H (\%) \\
\hline Ch $3\left(P_{1}\right)$ & 0.993 & 0.817 & 0.905 & & $P_{2} \times P_{9}$ & 5.247 & 4.367 & 4.807 & 2.2 \\
\hline Ch $8\left(P_{2}\right)$ & 1.605 & 1.587 & 1.596 & & $P_{3} \times P_{4}$ & 4.113 & 3.000 & 3.557 & $-38.2^{*}$ \\
\hline Ch $14\left(P_{3}\right)$ & 2.390 & 2.283 & 2.337 & & $\mathbf{P}_{3} \times \mathbf{P}_{5}$ & 2.007 & 1.693 & 1.850 & -28.9 \\
\hline Ch $16\left(P_{4}\right)$ & 5.920 & 5.583 & 5.752 & & $\mathbf{P}_{3} \times \mathbf{P}_{6}$ & 1.348 & 1.718 & 1.533 & $-34.5^{\star}$ \\
\hline Ch $18\left(P_{5}\right)$ & 2.705 & 2.500 & 2.603 & & $\mathbf{P}_{3} \times \mathbf{P}_{7}$ & 3.663 & 3.367 & 3.515 & $-41.4^{*}$ \\
\hline Ch $21\left(P_{6}\right)$ & 1.874 & 1.767 & 1.820 & & $\mathbf{P}_{3} \times \mathbf{P}_{8}$ & 4.085 & 3.830 & 3.958 & $69.4^{*}$ \\
\hline Ch $22\left(P_{7}\right)$ & 6.368 & 5.633 & 6.001 & & $\mathbf{P}_{3} \times \mathbf{P}_{9}$ & 4.843 & 4.133 & 4.488 & -4.6 \\
\hline Ch $25\left(P_{8}\right)$ & 2.140 & 1.767 & 1.953 & & $P_{4} \times P_{5}$ & 2.183 & 2.315 & 2.249 & $-60.9^{*}$ \\
\hline $\begin{array}{l}\text { Tomato } \\
139\left(P_{9}\right)\end{array}$ & 5.024 & 4.383 & 4.704 & & $\mathbf{P}_{4} \times \mathbf{P}_{6}$ & 2.897 & 2.191 & 2.544 & $-55.8^{*}$ \\
\hline$P_{1} \times P_{2}$ & 3.516 & 1.247 & 2.381 & $49.2^{*}$ & $\mathbf{P}_{4} \times \mathbf{P}_{7}$ & 5.617 & 4.467 & 5.042 & $-15.9^{*}$ \\
\hline$P_{1} \times P_{3}$ & 4.161 & 3.067 & 3.614 & $54.6^{*}$ & $\mathbf{P}_{4} \times \mathbf{P}_{8}$ & 3.019 & 3.067 & 3.043 & $-47.1^{*}$ \\
\hline$P_{1} \times P_{4}$ & 6.500 & 3.898 & 5.199 & -9.6 & $\mathbf{P}_{4} \times \mathbf{P}_{9}$ & 5.771 & 3.336 & 4.554 & -20.8 \\
\hline$P_{1} \times P_{5}$ & 1.964 & 1.612 & 1.788 & $-31.3^{*}$ & $\mathbf{P}_{5} \times \mathbf{P}_{6}$ & 2.646 & 1.757 & 2.201 & -15.4 \\
\hline$P_{1} \times P_{6}$ & 1.726 & 1.491 & 1.609 & -11.7 & $\mathbf{P}_{5} \times \mathbf{P}_{7}$ & 6.066 & 5.369 & 5.718 & -4.7 \\
\hline$P_{1} \times P_{7}$ & 4.116 & 3.533 & 3.825 & $-36.3^{*}$ & $\mathbf{P}_{5} \times \mathrm{P}_{8}$ & 4.416 & 1.676 & 3.046 & 17.0 \\
\hline$P_{1} \times P_{8}$ & 2.454 & 1.973 & 2.214 & 13.4 & $P_{5} \times P_{9}$ & 4.414 & 4.730 & 4.572 & -2.8 \\
\hline$P_{1} \times P_{9}$ & 2.912 & 2.386 & 2.649 & $-43.7^{*}$ & $\mathbf{P}_{6} \times \mathbf{P}_{7}$ & 3.804 & 2.825 & 3.315 & $-44.8^{*}$ \\
\hline$P_{2} \times P_{3}$ & 2.941 & 1.778 & 2.359 & 0.9 & $P_{6} \times P_{8}$ & 2.566 & 1.370 & 1.968 & 0.8 \\
\hline$P_{2} \times P_{4}$ & 4.567 & 2.234 & 3.400 & $-40.9^{*}$ & $P_{6} \times P_{9}$ & 5.726 & 3.725 & 4.726 & 0.5 \\
\hline$P_{2} \times P_{5}$ & 1.877 & 1.463 & 1.670 & $-35.8^{*}$ & $\mathbf{P}_{7} \times \mathbf{P}_{8}$ & 6.096 & 3.967 & 5.032 & $-16.2^{*}$ \\
\hline$P_{2} \times P_{6}$ & 1.540 & 1.550 & 1.545 & -15.1 & $P_{7} \times P_{9}$ & 3.832 & 2.305 & 3.069 & $-48.9^{\star}$ \\
\hline$P_{2} \times P_{7}$ & 5.251 & 3.760 & 4.505 & $-24.9^{*}$ & $P_{8} \times P_{9}$ & 3.186 & 2.467 & 2.827 & $-39.9^{*}$ \\
\hline$P_{2} \times P_{8}$ & 2.380 & 2.297 & 2.339 & 19.8 & $\begin{array}{c}\text { Katalina- } \\
522\end{array}$ & 5.201 & 4.133 & 4.667 & \\
\hline LSD at $5 \%$ & 0.960 & 0.504 & 0.773 & & LSD at $5 \%$ & 0.960 & 0.504 & 0.773 & \\
\hline
\end{tabular}


Table 3. Mean performance of some cherry tomato genotypes and their $F_{1}$ 's and high parent heterosis $(H)$ for average fruit weight in the 2014/15 and 2015/16 winter plantings under greenhouse conditions.

\begin{tabular}{|c|c|c|c|c|c|c|c|c|c|}
\hline \multirow{2}{*}{ Genotype } & \multicolumn{4}{|c|}{ Average fruit weight (g) } & \multirow{2}{*}{ Genotype } & \multicolumn{4}{|c|}{ Average fruit weight (g) } \\
\hline & $2014 / 15$ & $2015 / 16$ & Mean & H (\%) & & $\overline{2014 / 15}$ & $2015 / 16$ & Mean & $\mathbf{H}(\%)$ \\
\hline Ch $3\left(P_{1}\right)$ & 10.1 & 10.1 & 10.1 & & $P_{2} \times P_{9}$ & 13.1 & 11.9 & 12.5 & -1.6 \\
\hline Ch $8\left(P_{2}\right)$ & 11.9 & 13.5 & 12.7 & & $\mathbf{P}_{3} \times \mathbf{P}_{4}$ & 14.1 & 14.5 & 14.3 & $-36.2^{*}$ \\
\hline Ch $14\left(P_{3}\right)$ & 22.0 & 22.7 & 22.4 & & $P_{3} \times P_{5}$ & 16.3 & 18.5 & 17.4 & $-22.3^{*}$ \\
\hline Ch $16\left(P_{4}\right)$ & 12.4 & 12.0 & 12.2 & & $P_{3} \times P_{6}$ & 21.5 & 19.9 & 20.7 & $-7.6^{*}$ \\
\hline Ch $18\left(P_{5}\right)$ & 20.7 & 20.0 & 20.3 & & $\mathbf{P}_{3} \times \mathbf{P}_{7}$ & 15.0 & 11.8 & 13.4 & $-40.2^{*}$ \\
\hline Ch $21\left(P_{6}\right)$ & 22.0 & 21.3 & 21.7 & & $\mathbf{P}_{3} \times \mathbf{P}_{8}$ & 12.9 & 12.5 & 12.7 & $-43.3^{*}$ \\
\hline Ch $22\left(P_{7}\right)$ & 12.6 & 12.7 & 12.7 & & $\mathbf{P}_{3} \times \mathbf{P}_{9}$ & 14.1 & 15.3 & 14.7 & $-34.4^{*}$ \\
\hline Ch $25\left(P_{8}\right)$ & 12.2 & 12.6 & 12.4 & & $\mathbf{P}_{4} \times \mathbf{P}_{5}$ & 14.5 & 12.7 & 13.6 & $-33.0^{*}$ \\
\hline Tomato $139\left(P_{9}\right)$ & 14.3 & 10.7 & 12.5 & & $\mathbf{P}_{4} \times \mathbf{P}_{6}$ & 17.1 & 16.5 & 16.8 & $-22.3^{*}$ \\
\hline$P_{1} \times P_{2}$ & 9.6 & 9.8 & 9.7 & $-23.6^{*}$ & $P_{4} \times P_{7}$ & 11.4 & 11.6 & 11.5 & -9.5 \\
\hline$P_{1} \times P_{3}$ & 11.4 & 11.0 & 11.2 & $-50.0^{*}$ & $\mathbf{P}_{4} \times \mathbf{P}_{8}$ & 12.1 & 10.8 & 11.5 & -7.3 \\
\hline $\mathbf{P}_{1} \times \mathbf{P}_{4}$ & 11.1 & 8.7 & 9.9 & $-18.9^{*}$ & $\mathbf{P}_{4} \times \mathbf{P}_{9}$ & 12.2 & 11.3 & 11.8 & -5.6 \\
\hline$P_{1} \times P_{5}$ & 11.6 & 11.9 & 11.7 & $-42.4^{*}$ & $\mathbf{P}_{5} \times \mathbf{P}_{6}$ & 22.7 & 15.9 & 19.3 & $-11.1^{*}$ \\
\hline$P_{1} \times P_{6}$ & 14.5 & 13.6 & 14.1 & $-35.0^{*}$ & $P_{5} \times P_{7}$ & 13.5 & 14.1 & 13.8 & $-32.0^{*}$ \\
\hline$P_{1} \times P_{7}$ & 11.1 & 11.3 & 11.2 & $-11.8^{*}$ & $P_{5} \times P_{8}$ & 13.7 & 11.4 & 12.6 & $-37.9^{*}$ \\
\hline$P_{1} \times P_{8}$ & 9.6 & 9.9 & 9.7 & $-21.8^{*}$ & $P_{5} \times P_{9}$ & 14.3 & 14.5 & 14.4 & $-29.0^{*}$ \\
\hline$P_{1} \times P_{9}$ & 13.3 & 12.5 & 12.9 & 3.2 & $\mathbf{P}_{6} \times \mathbf{P}_{7}$ & 18.9 & 16.2 & 17.6 & $-18.9^{*}$ \\
\hline$P_{2} \times P_{3}$ & 13.3 & 15.0 & 14.1 & $-37.9^{*}$ & $\mathbf{P}_{6} \times \mathbf{P}_{8}$ & 14.9 & 15.1 & 15.0 & $-30.9^{*}$ \\
\hline$P_{2} \times P_{4}$ & 13.7 & 12.3 & 13.0 & 2.4 & $P_{6} \times P_{9}$ & 17.3 & 18.1 & 17.7 & $-18.4^{*}$ \\
\hline$P_{2} \times P_{5}$ & 15.5 & 16.9 & 16.2 & $-20.2^{*}$ & $P_{7} \times P_{8}$ & 13.7 & 13.1 & 13.4 & 5.5 \\
\hline$P_{2} \times P_{6}$ & 15.7 & 12.5 & 14.1 & $-35.0^{*}$ & $\mathbf{P}_{7} \times \mathbf{P}_{9}$ & 12.6 & 11.7 & 12.1 & -4.7 \\
\hline$P_{2} \times P_{7}$ & 11.3 & 12.4 & 11.9 & -6.3 & $P_{8} \times P_{9}$ & 12.4 & 11.5 & 11.9 & -4.8 \\
\hline$P_{2} \times P_{8}$ & 12.1 & 10.5 & 11.3 & $-11.0^{*}$ & Katalina & 10.3 & 11.9 & 11.1 & \\
\hline LSD at $5 \%$ & 0.8 & 0.9 & 1.3 & & LSD at $5 \%$ & 0.8 & 0.9 & 1.3 & \\
\hline
\end{tabular}

\section{Fruit length}

Fruit length trait reflected a great variation among the evaluated genotypes (Table 4) with values, ranging from $22.1 \mathrm{~mm}$ to $43.9 \mathrm{~mm}$. The longest fruits were shown by the genotype Ch $21(43.9 \mathrm{~mm})$, followed by genotype Ch $14 \quad(31.3 \quad \mathrm{~mm})$ with significant differences between them. Regarding hybrids, the hybrid Ch $14 \times$ Ch 21 had the longest fruits $(32.6 \mathrm{~mm})$, followed by hybrid Ch $18 \times$ Ch 21 (30.5 mm) without significant differences between them and with significant differences from the check hybrid Katalina-522 $(25.6 \mathrm{~mm})$. With regard to heterosis, none of the evaluated crosses showed significant positive heterobeltiosis values for fruit length trait. These results disagree with those found by Salib (2012), who estimated heterobeltiosis in some studied crosses for fruit length trait. 
Table 4. Mean performance of some cherry tomato genotypes and their $F_{1}$ 's and high parent heterosis $(H)$ for fruit length in the 2014/15 and 2015/16 winter plantings under greenhouse conditions.

\begin{tabular}{|c|c|c|c|c|c|c|c|c|c|}
\hline \multirow{2}{*}{ Genotype } & \multicolumn{4}{|c|}{ Fruit length $(\mathrm{cm})$} & \multirow{2}{*}{ Genotype } & \multicolumn{4}{|c|}{ Fruit length $(\mathrm{cm})$} \\
\hline & $2014 / 15$ & $2015 / 16$ & Mean & $\mathrm{H}(\%)$ & & $2014 / 15$ & $2015 / 16$ & Mean & H (\%) \\
\hline Ch $3\left(P_{1}\right)$ & 22.1 & 22.1 & 22.1 & & $\mathbf{P}_{2 \times P_{9}}$ & 24.5 & 23.8 & 24.1 & -2.8 \\
\hline Ch $8\left(P_{2}\right)$ & 24.9 & 24.1 & 24.5 & & $\mathbf{P}_{3} \times \mathbf{P}_{4}$ & 26.8 & 25.0 & 25.9 & $-17.3^{*}$ \\
\hline Ch $14\left(P_{3}\right)$ & 31.5 & 31.1 & 31.3 & & $P_{3} \times P_{5}$ & 28.6 & 27.2 & 27.9 & $-10.9^{*}$ \\
\hline Ch $16\left(P_{4}\right)$ & 24.9 & 24.1 & 24.5 & & $\mathbf{P}_{3} \times \mathbf{P}_{6}$ & 33.4 & 31.8 & 32.6 & $-25.7^{*}$ \\
\hline Ch $18\left(P_{5}\right)$ & 31.3 & 30.4 & 30.8 & & $P_{3} \times P_{7}$ & 26.9 & 24.3 & 25.6 & $-18.2^{*}$ \\
\hline Ch $21\left(P_{6}\right)$ & 44.5 & 43.3 & 43.9 & & $\mathbf{P}_{3} \times \mathbf{P}_{8}$ & 27.2 & 26.0 & 26.6 & $-15.0^{*}$ \\
\hline Ch $22\left(P_{7}\right)$ & 25.0 & 22.9 & 23.9 & & $\mathbf{P}_{3} \times \mathbf{P}_{9}$ & 28.2 & 25.7 & 26.9 & $-14.1^{*}$ \\
\hline Ch $25\left(P_{8}\right)$ & 24.9 & 24.0 & 24.4 & & $\mathbf{P}_{4} \times \mathbf{P}_{5}$ & 25.4 & 24.5 & 25.0 & $-18.8^{*}$ \\
\hline $\begin{array}{l}\text { Tomato } 139 \\
\left(P_{9}\right)\end{array}$ & 25.4 & 24.2 & 24.8 & & $\mathbf{P}_{4} \times \mathbf{P}_{6}$ & 28.0 & 26.5 & 27.3 & $-37.8^{*}$ \\
\hline $\mathbf{P}_{1} \times \mathbf{P}_{2}$ & 23.8 & 23.2 & 23.5 & -4.1 & $\mathbf{P}_{4} \times \mathbf{P}_{7}$ & 24.2 & 23.7 & 24.0 & -2.0 \\
\hline$P_{1} \times P_{3}$ & 24.4 & 22.1 & 23.3 & $-25.6^{*}$ & $\mathbf{P}_{4} \times \mathbf{P}_{8}$ & 24.2 & 22.9 & 23.6 & -3.7 \\
\hline $\mathbf{P}_{1} \times \mathbf{P}_{4}$ & 24.0 & 22.5 & 23.2 & $-5.3^{*}$ & $\mathbf{P}_{4} \times \mathbf{P}_{9}$ & 24.9 & 22.8 & 23.9 & -3.6 \\
\hline$P_{1} \times P_{5}$ & 25.6 & 22.2 & 23.9 & $-22.0^{*}$ & $\mathbf{P}_{5} \times \mathbf{P}_{6}$ & 33.0 & 28.1 & 30.5 & $-30.5^{*}$ \\
\hline$P_{1} \times P_{6}$ & 26.9 & 24.6 & 25.7 & $-41.5^{\star}$ & $P_{5} \times P_{7}$ & 25.5 & 25.4 & 25.4 & $-17.5^{*}$ \\
\hline$P_{1} \times P_{7}$ & 24.0 & 23.2 & 23.6 & -1.3 & $P_{5} \times P_{8}$ & 25.7 & 24.0 & 24.9 & $-19.2^{*}$ \\
\hline$P_{1} \times P_{8}$ & 22.4 & 22.1 & 22.3 & $-8.6^{*}$ & $P_{5} \times P_{9}$ & 28.0 & 25.8 & 26.9 & $-12.7^{*}$ \\
\hline$P_{1} \times P_{9}$ & 25.1 & 25.2 & 25.2 & 1.6 & $P_{6} \times P_{7}$ & 29.9 & 26.7 & 28.3 & $-65.3^{*}$ \\
\hline $\mathrm{P}_{2} \times \mathrm{P}_{3}$ & 28.0 & 25.0 & 26.5 & $-15.3^{*}$ & $P_{6} \times P_{8}$ & 28.2 & 27.0 & 27.6 & $-37.1^{*}$ \\
\hline $\mathbf{P}_{2} \times \mathbf{P}_{4}$ & 25.1 & 24.7 & 24.9 & 1.6 & $\mathbf{P}_{6} \times \mathbf{P}_{9}$ & 29.8 & 27.6 & 28.7 & $-34.6^{*}$ \\
\hline$P_{2} \times P_{5}$ & 29.1 & 28.2 & 28.7 & $-6.8^{*}$ & $P_{7} \times P_{8}$ & 25.5 & 22.7 & 24.1 & -1.2 \\
\hline$P_{2} \times P_{6}$ & 27.6 & 24.7 & 26.1 & $-40.6^{*}$ & $\mathbf{P}_{7} \times \mathbf{P}_{9}$ & 25.6 & 23.4 & 24.5 & -1.2 \\
\hline$P_{2} \times P_{7}$ & 24.6 & 23.6 & 24.1 & -1.6 & $\mathbf{P}_{8} \times \mathbf{P}_{9}$ & 24.4 & 23.5 & 24.0 & -3.3 \\
\hline$P_{2} \times P_{8}$ & 23.9 & 23.7 & 23.8 & -2.9 & Katalina & 26.1 & 25.1 & 25.6 & \\
\hline LSD at $5 \%$ & 1.7 & 1.4 & 1.3 & & LSD at $5 \%$ & 1.7 & 1.4 & 1.3 & \\
\hline
\end{tabular}

\section{Fruit diameter}

Regarding fruit diameter (Table 5), the genotype Ch 14 showed the highest value (30.9 mm), meanwhile, the lowest value was recorded in the genotype Ch $3(23.1 \mathrm{~mm})$. All the studied hybrids showed fruit diameter values between these values. Concerning heterosis, none of the evaluated crosses showed significant positive heterosis for this trait. The hybrids Ch $8 \times$ Ch 16 and Ch 21x Ch 22 showed insignificant positive heterosis for this trait. These results disagree with those found by Salib (2012). 
Table 5. Mean performance of some cherry tomato genotypes and their $F_{1}$ 's and high parent heterosis $(H)$ for fruit diameter in the 2014/15 and 2015/16 winter plantings under greenhouse conditions.

\begin{tabular}{|c|c|c|c|c|c|c|c|c|c|}
\hline \multirow{2}{*}{ Genotype } & \multicolumn{4}{|c|}{ Fruit diameter (cm) } & \multirow{2}{*}{ Genotype } & \multicolumn{4}{|c|}{ Fruit diameter $(\mathrm{cm})$} \\
\hline & $2014 / 15$ & $2015 / 16$ & Mean & H (\%) & & $2014 / 15$ & $2015 / 16$ & Mean & H (\%) \\
\hline Ch $3\left(P_{1}\right)$ & 23.1 & 23.0 & 23.1 & & $P_{2} \times P_{9}$ & 25.9 & 24.1 & 25.0 & -4.2 \\
\hline Ch $8\left(P_{2}\right)$ & 26.3 & 25.9 & 26.1 & & $\mathbf{P}_{3} \times \mathbf{P}_{4}$ & 27.6 & 27.2 & 27.4 & $-11.3^{*}$ \\
\hline Ch $14\left(P_{3}\right)$ & 31.9 & 29.9 & 30.9 & & $P_{3} \times P_{5}$ & 28.2 & 26.4 & 27.3 & $-11.7^{\star}$ \\
\hline Ch $16\left(P_{4}\right)$ & 26.4 & 24.6 & 25.5 & & $P_{3} \times P_{6}$ & 29.8 & 27.3 & 28.6 & $-7.4^{*}$ \\
\hline Ch $18\left(P_{5}\right)$ & 30.8 & 29.8 & 30.3 & & $\mathbf{P}_{3} \times \mathbf{P}_{7}$ & 25.9 & 24.4 & 25.1 & $-18.8^{*}$ \\
\hline Ch $21\left(P_{6}\right)$ & 29.0 & 28.7 & 28.9 & & $\mathbf{P}_{3} \times \mathbf{P}_{8}$ & 26.6 & 25.5 & 26.0 & $-15.9^{*}$ \\
\hline Ch $22\left(P_{7}\right)$ & 26.2 & 25.5 & 25.9 & & $P_{3} \times P_{9}$ & 26.9 & 27.0 & 26.9 & $-13.0^{*}$ \\
\hline Ch $25\left(P_{8}\right)$ & 25.5 & 25.3 & 25.4 & & $\mathbf{P}_{4} \times \mathbf{P}_{5}$ & 26.4 & 26.7 & 26.6 & $-12.2^{*}$ \\
\hline $\begin{array}{l}\text { Tomato } 139 \\
\left(P_{9}\right)\end{array}$ & 26.2 & 25.4 & 25.8 & & $P_{4} \times P_{6}$ & 28.5 & 19.4 & 23.9 & $-17.3^{*}$ \\
\hline $\mathbf{P}_{1} \times \mathbf{P}_{2}$ & 23.9 & 23.2 & 23.6 & $-9.6^{*}$ & $\mathbf{P}_{4} \times \mathbf{P}_{7}$ & 25.0 & 25.8 & 25.4 & -1.9 \\
\hline$P_{1} \times P_{3}$ & 24.9 & 24.7 & 24.8 & $-19.7^{*}$ & $\mathbf{P}_{4} \times \mathbf{P}_{8}$ & 25.9 & 23.7 & 24.8 & -2.8 \\
\hline$P_{1} \times P_{4}$ & 25.0 & 23.4 & 24.2 & -5.1 & $\mathbf{P}_{4} \times \mathbf{P}_{9}$ & 26.2 & 22.8 & 24.5 & -5.0 \\
\hline$P_{1} \times P_{5}$ & 25.9 & 23.4 & 24.7 & $-18.5^{\star}$ & $P_{5} \times P_{6}$ & 30.0 & 27.6 & 28.8 & -5.0 \\
\hline $\mathbf{P}_{1} \times \mathbf{P}_{6}$ & 26.6 & 23.3 & 25.0 & $-13.5^{\star}$ & $\mathbf{P}_{5} \times \mathbf{P}_{7}$ & 26.9 & 28.0 & 27.5 & $-9.2^{*}$ \\
\hline$P_{1} \times P_{7}$ & 25.0 & 24.2 & 24.6 & -5.0 & $P_{5} \times P_{8}$ & 25.2 & 24.1 & 24.7 & $-18.5^{\star}$ \\
\hline$P_{1} \times P_{8}$ & 24.0 & 24.2 & 24.1 & -5.1 & $P_{5} \times P_{9}$ & 28.1 & 27.3 & 27.7 & $-8.6^{*}$ \\
\hline$P_{1} \times P_{9}$ & 25.7 & 24.1 & 24.9 & -3.5 & $P_{6} \times P_{7}$ & 29.7 & 29.2 & 29.4 & 1.7 \\
\hline$P_{2} \times P_{3}$ & 28.0 & 24.8 & 26.4 & $-14.6^{*}$ & $P_{6} \times P_{8}$ & 27.7 & 27.0 & 27.4 & -5.2 \\
\hline$P_{2} \times P_{4}$ & 27.2 & 25.5 & 26.4 & 1.2 & $P_{6} \times P_{9}$ & 29.4 & 28.3 & 28.9 & 0.0 \\
\hline$P_{2} \times P_{5}$ & 29.2 & 27.0 & 28.1 & -7.3 & $P_{7} \times P_{8}$ & 26.5 & 24.4 & 25.5 & -1.5 \\
\hline$P_{2} \times P_{6}$ & 27.9 & 25.6 & 26.8 & -7.3 & $\mathrm{P}_{7} \times \mathrm{P}_{9}$ & 25.9 & 24.6 & 25.2 & -2.7 \\
\hline$P_{2} \times P_{7}$ & 25.9 & 25.7 & 25.8 & $-1.2^{*}$ & $P_{8} \times P_{9}$ & 25.5 & 23.4 & 24.5 & -5.0 \\
\hline$P_{2} \times P_{8}$ & 24.8 & 22.8 & 23.8 & -8.8 & Katalina & 25.8 & 24.9 & 25.3 & \\
\hline LSD at $5 \%$ & 1.4 & 3.7 & 2.2 & & LSD at $5 \%$ & 1.4 & 3.7 & 2.2 & \\
\hline
\end{tabular}

\section{Fruit firmness}

Data of fruit firmness are presented in Table 6. Combined data showed significant differences for this character among the evaluated genotypes. They ranged from 439.3 to $658.5 \mathrm{~g} / \mathrm{cm}^{2}$. The genotype Ch 3 , significantly, had the highest fruit firmness among all evaluated genotypes. For hybrids, the check hybrid Katalina-522, significantly, had the highest fruit firmness among all evaluated genotypes, followed by hybrid $\mathrm{Ch}$ $16 \times$ Ch $25\left(628.7 \mathrm{~g} / \mathrm{cm}^{2}\right)$ with significant differences between them. The lowest fruit firmness value was found in fruits of the hybrid Ch $22 \times$ Ch $25\left(439.3 \mathrm{~g} / \mathrm{cm}^{2}\right)$. With respect to heterosis, six out of the 36 evaluated hybrids exhibited significant positive heterosis for fruit firmness ranged from $4.2 \%$ for the hybrid Ch $21 \times$ Ch 22 to $11.1 \%$ for the hybrid Ch $16 \times$ Ch 25 . These results are in agreement with those of Salib (2012), Mahmoud and El-Eslamboly (2014), Renuka et al (2015) and Renuka and Sadashiva (2016) who found positive heterosis over better parent for this trait. Also, Khereba et al (2011) estimated heterosis over standard hybrid on cherry tomato for fruit firmness character. 
Table 6. Mean performance of some cherry tomato genotypes and their $F_{1}$ 's and high parent heterosis $(\mathrm{H})$ for fruit firmness in the 2014/15 and 2015/16 winter plantings under greenhouse conditions.

\begin{tabular}{|c|c|c|c|c|c|c|c|c|c|}
\hline \multirow{2}{*}{ Genotype } & \multicolumn{4}{|c|}{ Fruit firmness $\left(\mathrm{g} / \mathrm{cm}^{2}\right)$} & \multirow{2}{*}{ Genotype } & \multicolumn{4}{|c|}{ Fruit firmness $\left(\mathrm{g} / \mathrm{cm}^{2}\right)$} \\
\hline & 2014/152 & 015/16 & Mean & $\mathrm{H}(\%)$ & & 2014/15 & 2015/16 & Mean & $H(\%)$ \\
\hline Ch $3\left(P_{1}\right)$ & 660.0 & 657.0 & 658.5 & & $P_{2} \times P_{9}$ & 591.0 & 616.0 & 603.5 & 0.3 \\
\hline Ch $8\left(P_{2}\right)$ & 612.7 & 590.7 & 601.7 & & $\mathbf{P}_{3} \times \mathbf{P}_{4}$ & 502.0 & 567.0 & 534.5 & $-10.7^{*}$ \\
\hline Ch $14\left(P_{3}\right)$ & 600.0 & 596.7 & 598.3 & & $P_{3} \times P_{5}$ & 555.0 & 562.0 & 558.5 & $-6.7^{*}$ \\
\hline Ch $16\left(P_{4}\right)$ & 570.0 & 562.0 & 566.0 & & $\mathbf{P}_{3} \times \mathbf{P}_{6}$ & 592.0 & 614.3 & 603.2 & 0.8 \\
\hline Ch $18\left(P_{5}\right)$ & 594.0 & 589.3 & 591.7 & & $\mathbf{P}_{3} \times \mathbf{P}_{7}$ & 552.0 & 585.0 & 568.5 & $-5.0^{*}$ \\
\hline Ch $21\left(P_{6}\right)$ & 601.0 & 587.7 & 594.3 & & $\mathbf{P}_{3} \times \mathbf{P}_{8}$ & 516.7 & 554.7 & 535.7 & $-10.6^{*}$ \\
\hline Ch $22\left(P_{7}\right)$ & 571.0 & 582.7 & 576.8 & & $P_{3} \times P_{9}$ & 535.0 & 533.3 & 534.2 & $-10.7^{\star}$ \\
\hline Ch $25\left(P_{8}\right)$ & 505.3 & 518.0 & 511.7 & & $\mathbf{P}_{4} \times \mathbf{P}_{5}$ & 571.0 & 572.7 & 571.8 & $-3.4^{*}$ \\
\hline $\begin{array}{l}\text { Tomato } 139 \\
\left(P_{9}\right)\end{array}$ & 548.0 & 572.3 & 560.2 & & $\mathbf{P}_{4} \times \mathbf{P}_{6}$ & 601.0 & 638.3 & 619.7 & $4.3^{*}$ \\
\hline$P_{1} \times P_{2}$ & 483.0 & 492.0 & 487.5 & $-25.7^{*}$ & $\mathbf{P}_{4} \times \mathbf{P}_{7}$ & 540.0 & 550.3 & 545.2 & $-5.5^{\star}$ \\
\hline$P_{1} \times P_{3}$ & 524.0 & 541.7 & 532.8 & $-19.1^{*}$ & $P_{4} \times P_{8}$ & 616.0 & 641.3 & 628.7 & $11.1^{*}$ \\
\hline $\mathbf{P}_{1} \times \mathbf{P}_{4}$ & 600.0 & 597.0 & 598.5 & $-9.1^{*}$ & $\mathbf{P}_{4} \times \mathbf{P}_{9}$ & 592.0 & 639.0 & 615.5 & $8.8^{*}$ \\
\hline $\mathbf{P}_{1} \times \mathbf{P}_{5}$ & 556.0 & 571.3 & 563.7 & $-14.4^{*}$ & $P_{5} \times P_{6}$ & 593.0 & 642.7 & 617.8 & $4.0^{*}$ \\
\hline $\mathbf{P}_{1} \times \mathbf{P}_{6}$ & 529.0 & 541.7 & 535.3 & $-18.7^{*}$ & $P_{5} \times P_{7}$ & 541.0 & 555.3 & 548.2 & $-7.4^{\star}$ \\
\hline$P_{1} \times P_{7}$ & 562.0 & 574.3 & 568.2 & $-13.7^{*}$ & $P_{5} \times P_{8}$ & 551.0 & 551.3 & 551.2 & $-6.9^{*}$ \\
\hline$P_{1} \times P_{8}$ & 480.0 & 486.0 & 483.0 & $-26.7^{*}$ & $P_{5} \times P_{9}$ & 583.0 & 632.7 & 607.8 & 2.7 \\
\hline$P_{1} \times P_{9}$ & 580.0 & 600.0 & 590.0 & $-10.4^{*}$ & $P_{6} \times P_{7}$ & 616.0 & 622.7 & 619.3 & $4.2^{*}$ \\
\hline$P_{2} \times P_{3}$ & 533.3 & 576.7 & 555.0 & $-7.8^{*}$ & $P_{6} \times P_{8}$ & 543.0 & 570.3 & 556.7 & $-6.3^{*}$ \\
\hline$P_{2} \times P_{4}$ & 630.0 & 645.0 & 637.5 & $6.0^{*}$ & $P_{6} \times P_{9}$ & 600.0 & 615.3 & 607.7 & 2.3 \\
\hline$P_{2} \times P_{5}$ & 594.0 & 600.0 & 597.0 & -0.8 & $P_{7} \times P_{8}$ & 405.0 & 473.7 & 439.3 & $-23.8^{*}$ \\
\hline$P_{2} \times P_{6}$ & 555.0 & 563.7 & 559.3 & $-7.1^{*}$ & $P_{7} \times P_{9}$ & 513.0 & 541.7 & 527.3 & $-8.6^{*}$ \\
\hline$P_{2} \times P_{7}$ & 543.0 & 543.3 & 543.2 & $-9.7^{*}$ & $P_{8} \times P_{9}$ & 556.0 & 560.7 & 558.3 & -0.3 \\
\hline$P_{2} \times P_{8}$ & 503.0 & 560.0 & 531.5 & $-11.7^{*}$ & Katalina & 634.0 & 680.7 & 657.3 & \\
\hline LSD at $5 \%$ & 21.0 & 16.5 & 19.6 & & LSD at $5 \%$ & 21.0 & 16.5 & 19.6 & \\
\hline
\end{tabular}

\section{Fruit flesh thickness}

Combined analysis of both seasons showed significant differences for fruit flesh thickness among the evaluated genotypes (Table 7) ranging from 1.6 to $3.5 \mathrm{~mm}$. The genotype Ch 14 had the highest value (3.5 $\mathrm{mm}$ ) followed by the genotype Ch 21 (3.4 $\mathrm{mm}$ ) without significant differences between them. The hybrids Ch $14 \times$ Ch 21 and Ch 21 $\times$ Ch 22 had the highest value of fruit flesh thickness $(3.4 \mathrm{~mm})$ with significant differences with check hybrid Katalina-522 $(2.6 \mathrm{~mm})$. Concerning heterosis, only the cross Ch $3 \times$ Tomato 139 showed significant positive heterosis (8.0\%). These results partially agree with those found by Pujer et al (2014), Renuka et al (2015) and Renuka and Sadashiva (2016) who found positive heterosis over high parent for this trait. Also, Khereba et al (2011) estimated heterosis over standard hybrid on cherry tomato for fruit flesh thickness character. 
Table 7. Mean performance of some cherry tomato genotypes and their $F_{1}$ 's and high parent heterosis $(H)$ for fruit flesh thickness in the 2014/15 and 2015/16 winter plantings under greenhouse conditions.

\begin{tabular}{|c|c|c|c|c|c|c|c|c|c|}
\hline \multirow{3}{*}{\begin{tabular}{|l} 
Genotype \\
Ch $3\left(P_{1}\right)$ \\
\end{tabular}} & \multicolumn{4}{|c|}{ Fruit flesh thickness (mm) } & \multirow{3}{*}{$\begin{array}{l}\text { Genotype } \\
\mathrm{P}_{2} \times \mathrm{P}_{9}\end{array}$} & \multicolumn{4}{|c|}{ Fruit flesh thickness (mm) } \\
\hline & \multicolumn{2}{|c|}{ 2014/152015/16 } & \multirow{2}{*}{$\begin{array}{c}\text { Mean } \\
2.1\end{array}$} & \multirow[t]{2}{*}{ H (\%) } & & \multicolumn{4}{|c|}{ 2014/15 2015/16 Mean H(\%) } \\
\hline & 2.2 & 2.1 & & & & 2.4 & 2.3 & 2.3 & $-8.0^{\star}$ \\
\hline Ch $8\left(P_{2}\right)$ & 2.5 & 2.5 & 2.5 & & $\mathbf{P}_{3} \times \mathbf{P}_{4}$ & 3.0 & 2.8 & 2.9 & $-17.1^{*}$ \\
\hline Ch $14\left(P_{3}\right)$ & 3.5 & 3.4 & 3.5 & & $P_{3} \times P_{5}$ & 3.0 & 2.7 & 2.8 & $-20.0^{*}$ \\
\hline Ch $16\left(P_{4}\right)$ & 2.7 & 2.7 & 2.7 & & $P_{3} \times P_{6}$ & 3.5 & 3.3 & 3.4 & -2.9 \\
\hline Ch $18\left(P_{5}\right)$ & 3.3 & 3.1 & 3.2 & & $P_{3} \times P_{7}$ & 2.8 & 2.7 & 2.7 & $-22.9^{*}$ \\
\hline Ch $21\left(P_{6}\right)$ & 3.5 & 3.4 & 3.4 & & $P_{3} \times P_{8}$ & 2.0 & 1.9 & 1.9 & $-45.7^{*}$ \\
\hline Ch $22\left(P_{7}\right)$ & 2.4 & 2.4 & 2.4 & & $P_{3} \times P_{9}$ & 3.1 & 2.7 & 2.9 & $-17.2^{*}$ \\
\hline Ch $25\left(P_{8}\right)$ & 2.4 & 2.3 & 2.4 & & $P_{4} \times P_{5}$ & 3.0 & 2.8 & 2.9 & $-9.4^{*}$ \\
\hline Tomato $139\left(P_{9}\right)$ & 2.6 & 2.4 & 2.5 & & $P_{4} \times P_{6}$ & 3.4 & 3.2 & 3.3 & -2.9 \\
\hline$P_{1} \times P_{2}$ & 2.4 & 2.3 & 2.3 & $-8.0^{*}$ & $P_{4} \times P_{7}$ & 2.2 & 2.3 & 2.3 & $-14.8^{*}$ \\
\hline $\mathbf{P}_{1} \times \mathbf{P}_{3}$ & 2.3 & 2.1 & 2.2 & $-37.1^{*}$ & $P_{4} \times P_{8}$ & 2.4 & 2.2 & 2.3 & $-14.8^{*}$ \\
\hline$P_{1} \times P_{4}$ & 2.4 & 2.1 & 2.3 & $-14.8^{*}$ & $P_{4} \times P_{9}$ & 2.6 & 2.2 & 2.4 & $-11.1^{*}$ \\
\hline$P_{1} \times P_{5}$ & 2.3 & 2.2 & 2.2 & $-31.3^{*}$ & $P_{5} \times P_{6}$ & 3.6 & 2.3 & 3.0 & $-11.8^{*}$ \\
\hline$P_{1} \times P_{6}$ & 2.4 & 2.2 & 2.3 & $-32.4^{*}$ & $P_{5} \times P_{7}$ & 2.7 & 2.3 & 2.5 & $-21.9^{*}$ \\
\hline $\mathbf{P}_{1} \times \mathbf{P}_{7}$ & 2.4 & 2.3 & 2.3 & -4.2 & $P_{5} \times P_{8}$ & 2.2 & 2.1 & 2.2 & -31.3 \\
\hline$P_{1} \times P_{8}$ & 1.7 & 1.6 & 1.6 & $-33.3^{*}$ & $P_{5} \times P_{9}$ & 3.2 & 2.6 & 2.9 & $-9.4^{*}$ \\
\hline $\mathbf{P}_{1} \times \mathbf{P}_{9}$ & 2.7 & 2.7 & 2.7 & $8.0^{*}$ & $P_{6} \times P_{7}$ & 3.4 & 3.5 & 3.4 & 0.0 \\
\hline $\mathbf{P}_{2} \times \mathbf{P}_{3}$ & 2.4 & 2.0 & 2.2 & $-37.1^{*}$ & $P_{6} \times P_{8}$ & 2.4 & 2.2 & 2.3 & $-32.4^{*}$ \\
\hline$P_{2} \times P_{4}$ & 2.8 & 2.3 & 2.5 & $-7.4^{*}$ & $P_{6} \times P_{9}$ & 3.4 & 3.2 & 3.3 & -2.9 \\
\hline$P_{2} \times P_{5}$ & 2.9 & 2.8 & 2.9 & $-9.4^{*}$ & $\mathbf{P}_{7} \times \mathbf{P}_{8}$ & 2.2 & 2.2 & 2.2 & $-8.3^{\star}$ \\
\hline $\mathbf{P}_{2} \times \mathbf{P}_{6}$ & 2.8 & 2.6 & 2.7 & $-20.6^{*}$ & $P_{7} \times P_{9}$ & 2.4 & 2.3 & 2.3 & $-8.0^{*}$ \\
\hline $\mathbf{P}_{2} \times \mathbf{P}_{7}$ & 2.4 & 2.3 & 2.3 & $-8.0^{*}$ & $P_{8} \times P_{9}$ & 2.6 & 2.4 & 2.5 & 0.0 \\
\hline$P_{2} \times P_{8}$ & 2.2 & 1.9 & 2.0 & $-20.0^{*}$ & Katalina & 2.8 & 2.4 & 2.6 & \\
\hline LSD at $5 \%$ & 0.1 & 0.2 & 0.2 & & LSD at $5 \%$ & 0.1 & 0.2 & 0.2 & \\
\hline
\end{tabular}

\section{Total soluble solids (TSS)}

Data of fruit total soluble solids are presented in Table 8. Combined analysis showed significant differences for this character, ranging from $5.1 \%$ to $7.7 \%$. The highest TSS value was detected in fruits of the hybrid Ch $16 \times$ Tomato $139(7.7 \%)$, followed by the hybrids Ch $14 \times$ Ch 22 and Ch $16 \times$ Ch 22 (7.5\%) without significant differences between them, but with significant differences with check hybrid Katalina-522 (6.4\%). The genotype Ch 18 had the lowest TSS value (5.1\%). Regarding heterosis, 14 crosses out of the 36 evaluated ones exhibited significant positive heterosis for fruit TSS ranged from $6.0 \%$ for the hybrid Ch $22 \times$ Tomato 139 to $13.6 \%$ for the hybrid Ch $14 \times$ Ch 22 . These results agree with those found by Fang et al (2002), Khereba et al (2011), Salib (2012) and Pujer et al (2014) who found positive heterosis over better parent and control hybrid on cherry tomato for this character. 
Table 8. Mean performance of some cherry tomato genotypes and their $F_{1}$ 's and high parent heterosis (H) for fruit TSS in the 2014/15 and 2015/16 winter plantings under greenhouse conditions.

\begin{tabular}{|c|c|c|c|c|c|c|c|c|c|}
\hline \multirow{2}{*}{ Genotype } & \multicolumn{4}{|c|}{ Fruit TSS (\%) } & \multirow{2}{*}{ Genotype } & \multicolumn{4}{|c|}{ Fruit TSS (\%) } \\
\hline & $2014 / 15$ & 2015/16 & Mean & H (\%) & & 2014/15 & 2015/16 & Mean & H (\%) \\
\hline Ch $3\left(P_{1}\right)$ & 5.8 & 5.7 & 5.7 & & $\mathbf{P}_{2} \times \mathbf{P}_{9}$ & 6.9 & 7.0 & 6.9 & 2.9 \\
\hline Ch $8\left(P_{2}\right)$ & 5.2 & 5.1 & 5.2 & & $\mathbf{P}_{3} \times \mathbf{P}_{4}$ & 6.1 & 6.9 & 6.5 & $-7.1^{*}$ \\
\hline Ch $14\left(P_{3}\right)$ & 5.4 & 5.8 & 5.6 & & $P_{3} \times P_{5}$ & 5.8 & 6.5 & 6.2 & $10.7^{*}$ \\
\hline Ch $16\left(P_{4}\right)$ & 6.2 & 7.7 & 7.0 & & $\mathbf{P}_{3} \times \mathbf{P}_{6}$ & 5.1 & 5.6 & 5.3 & -5.4 \\
\hline Ch $18\left(P_{5}\right)$ & 5.0 & 5.2 & 5.1 & & $\mathbf{P}_{3} \times \mathbf{P}_{7}$ & 7.3 & 7.6 & 7.5 & $13.6^{*}$ \\
\hline Ch $21\left(P_{6}\right)$ & 5.0 & 5.5 & 5.2 & & $\mathbf{P}_{3} \times \mathbf{P}_{8}$ & 5.8 & 6.4 & 6.1 & -4.7 \\
\hline Ch $22\left(P_{7}\right)$ & 6.7 & 6.6 & 6.6 & & $\mathbf{P}_{3} \times \mathbf{P}_{9}$ & 6.8 & 7.5 & 7.2 & $7.5^{\star}$ \\
\hline Ch $25\left(P_{8}\right)$ & 6.4 & 6.3 & 6.4 & & $\mathbf{P}_{4} \times \mathbf{P}_{5}$ & 6.0 & 8.0 & 7.0 & 0.0 \\
\hline Tomato $139\left(P_{9}\right)$ & 6.5 & 6.9 & 6.7 & & $\mathbf{P}_{4} \times \mathbf{P}_{6}$ & 6.0 & 7.0 & 6.5 & $-7.1^{*}$ \\
\hline $\mathbf{P}_{1} \times \mathbf{P}_{2}$ & 6.1 & 6.2 & 6.2 & $8.8^{*}$ & $\mathbf{P}_{4} \times \mathbf{P}_{7}$ & 6.9 & 8.1 & 7.5 & $7.1^{*}$ \\
\hline$P_{1} \times P_{3}$ & 6.3 & 6.6 & 6.4 & $12.3^{*}$ & $\mathbf{P}_{4} \times \mathbf{P}_{8}$ & 6.3 & 7.6 & 6.9 & -1.4 \\
\hline$P_{1} \times P_{4}$ & 6.4 & 6.6 & 6.5 & -7.1 & $\mathbf{P}_{4} \times \mathbf{P}_{9}$ & 7.3 & 8.0 & 7.7 & $10.0^{*}$ \\
\hline$P_{1} \times P_{5}$ & 5.9 & 5.9 & 5.9 & 3.5 & $\mathbf{P}_{5} \times \mathbf{P}_{6}$ & 5.3 & 5.7 & 5.5 & 5.8 \\
\hline$P_{1} \times P_{6}$ & 5.5 & 5.4 & 5.4 & -5.3 & $\mathbf{P}_{5} \times \mathbf{P}_{7}$ & 6.4 & 7.6 & 7.0 & $6.1^{*}$ \\
\hline$P_{1} \times P_{7}$ & 7.0 & 7.0 & 7.0 & $6.1^{*}$ & $\mathbf{P}_{5} \times \mathbf{P}_{8}$ & 5.8 & 6.5 & 6.1 & -4.7 \\
\hline$P_{1} \times P_{8}$ & 6.1 & 6.3 & 6.2 & -3.1 & $P_{5} \times P_{9}$ & 6.7 & 7.9 & 7.3 & $8.1^{*}$ \\
\hline$P_{1} \times P_{9}$ & 6.5 & 8.0 & 7.2 & $7.5^{\star}$ & $P_{6} \times P_{7}$ & 6.1 & 7.0 & 6.6 & 0.0 \\
\hline$P_{2} \times P_{3}$ & 6.0 & 6.4 & 6.2 & $10.7^{*}$ & $P_{6} \times P_{8}$ & 5.6 & 5.8 & 5.7 & $-10.9^{*}$ \\
\hline $\mathbf{P}_{2} \times \mathbf{P}_{4}$ & 5.5 & 6.2 & 5.9 & $-15.7^{\star}$ & $\mathbf{P}_{6} \times \mathbf{P}_{9}$ & 6.1 & 6.7 & 6.4 & -4.5 \\
\hline$P_{2} \times P_{5}$ & 5.4 & 5.7 & 5.5 & 5.8 & $\mathbf{P}_{7} \times \mathbf{P}_{8}$ & 6.5 & 6.9 & 6.7 & 1.5 \\
\hline $\mathbf{P}_{2} \times \mathbf{P}_{6}$ & 6.0 & 5.5 & 5.8 & $11.5^{*}$ & $\mathbf{P}_{7} \times \mathbf{P}_{9}$ & 6.5 & 7.6 & 7.1 & $6.0^{*}$ \\
\hline $\mathbf{P}_{2} \times \mathbf{P}_{7}$ & 6.6 & 7.0 & 6.8 & 3.0 & $P_{8} \times P_{9}$ & 6.5 & 7.3 & 6.9 & 3.0 \\
\hline$P_{2} \times P_{8}$ & 5.3 & 5.9 & 5.6 & $-12.5^{\star}$ & Katalina & 6.5 & 6.3 & 6.4 & \\
\hline LSD at $5 \%$ & 0.2 & 0.3 & 0.4 & & LSD at $5 \%$ & 0.2 & 0.3 & 0.4 & \\
\hline
\end{tabular}

\section{Ascorbic acid content}

Combined analysis of both seasons showed significant differences for ascorbic acid content (vitamin C) character among the evaluated genotypes (Table 9), ranging from 10.5 to $21.8 \mathrm{mg} / 100 \mathrm{~g}$ fresh fruit. Fruits of the genotypes Ch 16 and $\mathrm{Ch} 3$ had the highest ascorbic acid content (19.5 and 19.3 $\mathrm{mg} / 100 \mathrm{~g}$ fresh fruit, respectively) without significant differences between them. For hybrids, Fruits of the check hybrid Katalina522 had the highest ascorbic acid content $(21.8 \mathrm{mg} / 100 \mathrm{~g}$ fresh fruit), followed by the hybrids Ch $3 \times$ Ch 16, Ch $3 \times$ Ch 22 and Ch
$8 \times$ Ch $22(19.0 \mathrm{mg} / 100 \mathrm{~g}$ fresh fruit) with significant differences between them. The lowest value of ascorbic acid content was recorded in fruits of the genotype Ch 21 $(10.5 \mathrm{mg} / 100 \mathrm{~g}$ fresh fruit). With regard to heterosis, 9 out of the 36 evaluated hybrids showed significant positive heterosis for this trait, ranging from $3.7 \%$ for the hybrid Ch $8 \times$ Ch 25 to $25.0 \%$ for the hybrid Ch $8 \times$ Ch 18 . Fang et al (2002), Khereba et al (2011) Pujer et al (2014) and Mahmoud and ElEslamboly (2014) showed positive heterosis over the high parent and control for ascorbic acid content trait. 
Table 9. Mean performance of some cherry tomato genotypes and their $F_{1}$ 's and high parent heterosis (H) for fruit ascorbic acid content in the 2014/15 and 2015/16 winter plantings under greenhouse conditions.

\begin{tabular}{|c|c|c|c|c|c|c|c|c|c|}
\hline \multirow[t]{2}{*}{ Genotype } & \multicolumn{4}{|c|}{$\begin{array}{l}\text { Fruit ascorbic acid content } \\
\text { (mg/100 } \mathrm{g} \text { fresh fruit) }\end{array}$} & \multirow[t]{2}{*}{ Genotype } & \multicolumn{4}{|c|}{$\begin{array}{l}\text { Fruit ascorbic acid content } \\
(\mathrm{mg} / 100 \mathrm{~g} \text { fresh fruit) }\end{array}$} \\
\hline & $2014 / 15$ & $2015 / 16$ & Mean & $\mathbf{H}(\%)$ & & $2014 / 15$ & $2015 / 16$ & Mean & $\mathbf{H}(\%)$ \\
\hline Ch $3\left(P_{1}\right)$ & 19.4 & 19.1 & 19.3 & & $P_{2} \times P_{9}$ & 16.7 & 15.7 & 16.2 & 1.3 \\
\hline Ch $8\left(P_{2}\right)$ & 14.7 & 14.2 & 14.4 & & $\mathbf{P}_{3} \times \mathbf{P}_{4}$ & 16.4 & 16.0 & 16.2 & $-16.9^{*}$ \\
\hline Ch $14\left(P_{3}\right)$ & 12.5 & 12.3 & 12.4 & & $\mathbf{P}_{3} \times \mathbf{P}_{5}$ & 13.0 & 12.6 & 12.8 & $-7.9^{*}$ \\
\hline Ch $16\left(P_{4}\right)$ & 19.9 & 19.1 & 19.5 & & $P_{3} \times P_{6}$ & 11.7 & 11.0 & 11.4 & $-8.1^{*}$ \\
\hline Ch $18\left(P_{5}\right)$ & 14.1 & 13.6 & 13.9 & & $\mathbf{P}_{3} \times \mathbf{P}_{7}$ & 15.2 & 14.6 & 14.9 & $-3.3^{*}$ \\
\hline Ch $21\left(P_{6}\right)$ & 10.7 & 10.3 & 10.5 & & $\mathbf{P}_{3} \times \mathbf{P}_{8}$ & 13.0 & 12.6 & 12.8 & $-21.5^{\star}$ \\
\hline Ch $22\left(P_{7}\right)$ & 15.5 & 15.2 & 15.4 & & $P_{3} \times P_{9}$ & 17.1 & 16.5 & 16.8 & $5.0^{*}$ \\
\hline Ch $25\left(P_{8}\right)$ & 16.5 & 16.1 & 16.3 & & $\mathbf{P}_{4} \times \mathbf{P}_{5}$ & 12.6 & 12.2 & 12.4 & $-36.4^{*}$ \\
\hline Tomato $139\left(\mathrm{P}_{\mathrm{g}}\right)$ & 16.2 & 15.9 & 16.0 & & $\mathbf{P}_{4} \times \mathbf{P}_{6}$ & 14.7 & 14.0 & 14.4 & $-26.2^{*}$ \\
\hline$P_{1} \times P_{2}$ & 11.4 & 11.1 & 11.3 & $-41.5^{\star}$ & $\mathbf{P}_{4} \times \mathbf{P}_{7}$ & 14.7 & 14.0 & 14.4 & $-26.1^{*}$ \\
\hline$P_{1} \times P_{3}$ & 16.2 & 15.8 & 16.0 & $-17.1^{*}$ & $\mathbf{P}_{4} \times \mathbf{P}_{8}$ & 16.7 & 16.4 & 16.6 & $-14.9^{*}$ \\
\hline$P_{1} \times P_{4}$ & 19.2 & 18.8 & 19.0 & $-2.6^{*}$ & $\mathbf{P}_{4} \times \mathbf{P}_{9}$ & 14.0 & 13.4 & 13.7 & $-29.7^{*}$ \\
\hline$P_{1} \times P_{5}$ & 16.6 & 16.3 & 16.4 & $-15.0^{*}$ & $\mathbf{P}_{5} \times \mathbf{P}_{6}$ & 17.8 & 16.4 & 17.1 & $23.0^{*}$ \\
\hline$P_{1} \times P_{6}$ & 11.5 & 11.0 & 11.3 & $-41.5^{\star}$ & $P_{5} \times P_{7}$ & 19.2 & 18.6 & 18.9 & $22.7^{*}$ \\
\hline$P_{1} \times P_{7}$ & 19.5 & 18.4 & 19.0 & -1.6 & $P_{5} \times P_{8}$ & 16.7 & 16.5 & 16.6 & 1.8 \\
\hline$P_{1} \times P_{8}$ & 16.0 & 15.6 & 15.8 & $-18.1^{*}$ & $\mathbf{P}_{5} \times \mathbf{P}_{9}$ & 17.5 & 16.6 & 17.1 & $6.9^{*}$ \\
\hline$P_{1} \times P_{9}$ & 14.4 & 13.9 & 14.2 & $-26.4^{*}$ & $P_{6} \times P_{7}$ & 14.2 & 14.1 & 14.2 & $-7.8^{*}$ \\
\hline$P_{2} \times P_{3}$ & 15.1 & 14.6 & 14.8 & 2.8 & $P_{6} \times P_{8}$ & 12.5 & 12.4 & 12.4 & $-23.9^{*}$ \\
\hline$P_{2} \times P_{4}$ & 18.6 & 18.2 & 18.4 & $-5.6^{\star}$ & $P_{6} \times P_{9}$ & 16.4 & 15.8 & 16.1 & 0.6 \\
\hline$P_{2} \times P_{5}$ & 18.4 & 17.7 & 18.0 & $25.0^{*}$ & $P_{7} \times P_{8}$ & 14.2 & 13.5 & 13.9 & $-14.7^{\star}$ \\
\hline $\mathbf{P}_{2} \times \mathbf{P}_{6}$ & 12.0 & 11.8 & 11.9 & $-17.4^{*}$ & $\mathbf{P}_{7} \times \mathbf{P}_{9}$ & 19.1 & 18.6 & 18.9 & $18.1^{*}$ \\
\hline$P_{2} \times P_{7}$ & 19.3 & 18.6 & 19.0 & $23.4^{*}$ & $P_{8} \times P_{9}$ & 18.1 & 17.4 & 17.7 & $8.6^{*}$ \\
\hline$P_{2} \times P_{8}$ & 17.2 & 16.6 & 16.9 & $3.7^{*}$ & Katalina & 22.1 & 21.4 & 21.8 & \\
\hline LSD at $5 \%$ & 0.8 & 0.3 & 0.5 & & LSD at $5 \%$ & 0.8 & 0.3 & 0.5 & \\
\hline
\end{tabular}

\section{CONCLUSION}

From this study, it can be concluded that the crosses Ch $3 \times$ Ch 16, Ch $16 \times$ Ch 22, Ch $18 \times$ Ch 22 and Ch $22 \times$ Ch 25 were the best hybrids with respect to yield and reasonable fruit characters.

\section{ACKNOWLEDGEMENT}

The author expresses his deepest appreciation to project Improving the Main Vegetable Crops and Hybrid Production for continuous help and support throughout this study. 


\section{REFERENCES}

Abo-Hamda, E. M. E. H. (2012). Development and evaluation of some cherry tomato hybrids and genetic studies of some economic characters. Ph.D. Thesis, Fac. Agric., Cairo Univ., Egypt, $109 \mathrm{p}$.

Alarcon, J., M. Bolarin, M. Sanchez-Blanco and Torrecillas (1994). Growth, Yield and Water Relations of Normal Fruited and Cherry Tomato Cultivars Irrigated with Saline Water. J. Hort. Sci. 69 (2): 283288.

Anonymous (2009). Cherry tomato nutritional information; USDA National Nutritional Database for Standard Reference (www.lose-weightwithus.com/cherry tomatonutrition. html).

AOAC, Association of Official Agricultural Chemists (1990). Official Methods of Analysis. $15^{\text {th }}$ ed , Washington. D.C., USA.

Falconer, D. S. and T. F. C. Mackay (1996). Introduction to Quantitative Genetics $\left(4^{\text {th }}\right.$ edn), Longman, London.

Fang, L., Q. Zhang, Y. X. Dong, G. Y. Zhao and H. J. Liao. (2002). Selection of a new cherry tomato (Lycopersicon esculentum) $F_{1}$ hybrid Shengguo. China Vegetables (4): 25-27.

Fehr, W. R. (1987). Principles of Cultivar Development. Vol. I. MacMillan, USA.

Gomez, A. K. and A. A. Gomez (1984). Statistical Procedures for Agricultural Research. $2^{\text {nd }}$ ed. John Wiley and Sons, Inc., New York.

Kavitha, P., K. S. Shivashankara, V. K. Rao, A. T. Sadashiva, K. V. Ravishankar and G. J. Sathish (2014). Genotypic variability for antioxidant and quality parameters among tomato cultivars, hybrids, cherry tomatoes and wild species. J. Sci. Food Agric. 94: 993-999.

Khereba, A. H., K. E. A. Abdel-Ati, E. M. E. Khalil and E. M. I. Abo-Hamda (2011). Production and evaluation of some cherry tomato hybrids. Egypt. J. Hort. 38 (2): 169-188.

Mahmoud, A. M. A. and A. A. S. A. ElEslamboly (2014). Genetic analysis to find suitable parents for development of cherry tomato hybrids under green house conditions. Egypt. J. Plant Breed. 19 (1): 55-70.

Muttappanavar, R. D., A. T. Sadashiva, R. C. Vijendrakumar, B. N. Roopa and P. T. Vasantha (2014). Combining ability analysis of growth, yield and quality traits in cherry tomato (Solanum lycopersicum var. cerasiforme). Molec. Plant Breed. 5 (4): 18-23.

Pujer, P., R. C. Jagadeesha and B. Mahesh (2014). Combining ability analysis and heterosis for growth, yield and quality traits in cherry tomato (Solanum lycopersicum L. var. cerasiforme Mill). Inter. J. Appl. Agric. Hort. Sci. 5 (5): 758 761.

Renuka, D. M. and A. T. Sadashiva (2016). Heterosis for growth, yield and quality traits in cherry tomato (Solanum lycopersicum var. cerasiforme). Plant Archives 16 (2): 654-658.

Renuka, D. M., A. T. Sadashiva, B. T. Kavita, R. C. Vijendrakumar and M. R. Hanumanthiah (2014). Evaulation of cherry tomato lines (Solanumlycopersicum var. cerasiforme) for growth, yield and quality traits. Plant Archives 14 (1): 151-154.

Renuka D. M., T. H. Singh, S. V. Geeta and Sheela Malaghan (2015). Combining ability analysis of growth, yield and quality traits in cherry tomato (Solanum lycopersicum var. cersiforme). Inter. J. Adv. Res. 3 (7): 319-325.

Salib, F. S. (2012). Mode of inheritance and heterobeltiosis in tomato (lycopersicon esculentum mill). Egypt . J. Appl. Sci. 27 (7): 394-406.

Snedecor, G. W. and W. G. Cochran (1989). Statistical Methods. $8^{\text {th }}$ ed. Iowa State Univ. Press, Ames, lowa, USA. 


\title{
الإستفادة من قوة الهجين فى الطماطم الكريزية
}

\author{
أشرف عبدالله حامد \\ قسم تربية الخضر - معهد بحوث البساتين - مركز البحوث الزراعية - مصر
}

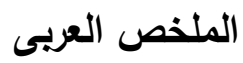

أجريت هذه الدراسة بمزرعة بحوث الخضر بقها بمحافظة القليوبيه التابعة لمركز البحوث الزراعية- مصر خلال الفترة من

2013 إلى 2016 لإنتاج بعض هجن الطماطم الكريزية وتقييمها تحت ظروف البيوت الدحمية. وقد نم تقييم 36 هجين

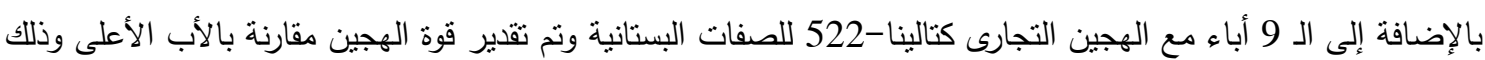

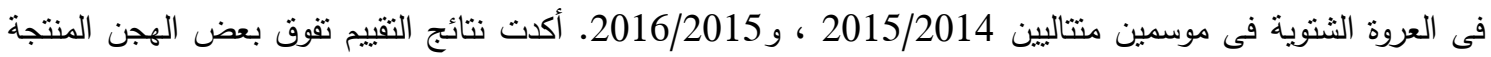

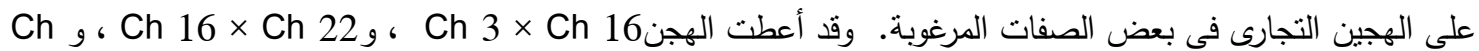
18 Ch 22 × و Ch 22 × Ch 25 أعلى محصول للنبات. كما أعطت بعض الهجن قوة هجين معنوية مقارنة بالأب

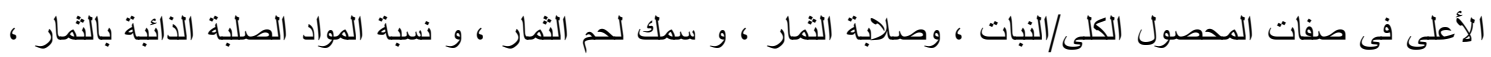

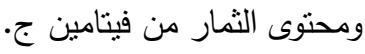


